\title{
Informalidad laboral y derecho a la salud en México, un análisis crítico
}

\author{
The informality of labor and the right to health in Mexico: \\ a critical analysis
}

Oswaldo Medina-Gómez (https://orcid.org/0000-0003-1286-7317) ${ }^{1}$

Oliva López-Arellano (https://orcid.org/0000-0001-8363-6888) ${ }^{2}$

\footnotetext{
${ }^{1}$ Unidad de Investigación en Epidemiología Clínica, HGR 1, Instituto Mexicano del Seguro Social. Gabriel Mancera 222, Del Valle, Benito Juárez. 03100 Ciudad de México México. epired@gmail.com

${ }^{2}$ Doctorado en Ciencias en Salud Colectiva, Universidad Autónoma Metropolitana. Cidade do México Distrito Federal México.
}

\begin{abstract}
In light of the labor conditions defined within the framework of neoliberalism implemented in Mexico, labor dynamics are problematized, the main problems of access to the health and social security system are identified and the systematic violation of rights - especially the right to work, to social security and to health - are discussed. The objective of this article is to contribute with proposals for the construction of a comprehensive, inclusive, healthcare and social security system that contributes to the improvement of the lives of workers.

Key words Labor, Informal workers, Right to health, Mexico
\end{abstract}

Resumen Frente a las condiciones de trabajo definidas en el marco del neoliberalismo implementado en México, se problematiza las dinámicas laborales, identifica los principales problemas de acceso al sistema de salud y de seguridad social $y$ discute la vulneración sistemática de derechos, principalmente del derecho al trabajo, a la seguridad social y a la salud. Se propone contribuir con propuestas para la construcción de un sistema de salud y seguridad social integral, incluyente y solidario que coadyuve a mejorar la vida de trabajadores y trabajadoras.

Palabras clave Trabajo, Trabajadores informales, Derecho a la salud, México 


\section{Introducción}

A partir de la década de los ochentas, México se incorporó a la modernidad neoliberal privilegiando un modelo de mercado abierto ${ }^{1,2}$.Esta incorporación reconfiguró las relaciones Estado-sociedad y Estado-mercado con un impacto negativo sobre la seguridad económica de amplios sectores de la población y sobre el conjunto de instituciones, normas, políticas, programas, servicios y recursos públicos empleados por el Estado para contribuir al ejercicio pleno de los derechos a la seguridad social, la salud, la educación, la vivienda y la alimentación, así como para la atención a poblaciones en situaciones de pobreza, vulnerabilidad o marginación que caracterizaban a un sistema de bienestar ${ }^{3}$.

El mercado de trabajo y las condiciones laborales en el país se modificaron afectando sustancialmente las relaciones contractuales entre trabajadores y patrones que progresivamente fueron vaciando de contenido la noción de "trabajo decente" como trabajo productivo, que genera valor para la sociedad, impulsa el desarrollo, es justamente remunerado, ejercido en condiciones de libertad, igualdad, seguridad y con dignidad. Reconocido como un derecho desde la Declaración Universal de los Derechos Humanos ${ }^{4}$, en el Pacto Internacional de los Derechos Económicos, Sociales y Culturales (PIDESC) en 1966, que define sus contenidos y las obligaciones jurídicas de los Estados parte en los artículos 6, 7, 8 y $9^{5}$ y en las Observaciones Generales 18 y 19 elaboradas por el Comité de Derechos Económicos, Sociales y Culturales ${ }^{6}$. La Constitución de los Estados Unidos Mexicanos en su artículo 123 establece el derecho al trabajo en términos de "Toda persona tiene derecho al trabajo digno y socialmente útil; al efecto se promoverán la creación de empleos y la organización social de trabajo conforme a la ley"7.

En los últimos 35 años en México se reduce y deteriora el empleo formal y se incrementa el trabajo precario (informal, temporal, sin contrato y sin seguridad social) como un mecanismo compensatorio frente a la reducción de puestos de trabajo formal. Si bien esta precarización laboral permite a las personas y a las familias obtener ingresos económicos para contender con sus necesidades más urgentes e incluso puede funcionar como un ingreso complementario ${ }^{8}$, en el país predomina como la principal fuente de recursos y la población ocupada en esta situación corresponde al 60 por ciento del total de la población trabajadora. En particular, la participación de las mujeres y jóvenes dentro del sector informal resulta ser un mecanismo compensatorio de la disminución del poder adquisitivo de las familias $\mathrm{y}$ el incremento en las tasas de desempleo que se presenta con mayor frecuencia en quienes cuentan con un nivel escolar de secundaria y más?.

La firma del Tratado de Libre Comercio con América del Norte (TLCAN) en 1994 acelera la flexibilización del trabajo en el país con propuestas de salarios por hora, simplificación de la conclusión de contratos, jornada laboral flexible y adecuación de prestaciones económicas a la capacidad de las empresas ${ }^{10}$. Para 2017, hay suficiente evidencia que la apertura de mercados y la liberalización de la economía contribuyó directamente a acentuar las desigualdades sociales, la concentración del ingreso, la caída vertiginosa de los salarios y la precarización del empleo existente ${ }^{3}$, con una vulneración sistemática del derecho al trabajo en el país.

Considerando lo anterior, el presente análisis problematiza las dinámicas laborales formal e informal, identifica los principales problemas de acceso al sistema de salud y de seguridad social y discute la vulneración sistemática de derechos, en particular del derecho al trabajo, a la seguridad social y a la salud, con la intención de contribuir con propuestas para la construcción de un sistema de salud y seguridad social integral, incluyente y solidario que coadyuve a mejorar la vida de trabajadores y trabajadoras.

\section{La precarización del trabajo y el impulso a la informalidad}

Durante la implementación del modelo neoliberal en México, se impulsó un régimen de dominación financiera sobre las políticas públicas, apertura y desregulación de los mercados de bienes y servicios e irrestricta movilidad del capital financiero para lo cual fue necesario subordinar en forma extrema el mundo del trabajo y en forma simultánea desmantelarel sistema de bienestar desarrollado previamente ${ }^{11}$, con el argumento de modernizar al país y fortalecer una economía abierta. Sin embargo, a 35 años de su implementación el modelo neoliberal, ha tenido un pobre efecto para impulsar la inversión productiva y erosiona a largo plazo las fuentes de crecimiento de la productividad, mientras que la frágil estabilidad económica se sustenta evitando el crecimiento en la tasa de salarios ${ }^{12}$. En el periodo 1980-2010 el salario mínimo pierde el 75 por ciento de su capacidad adquisitiva y el salario contractual se reduce en 45 por ciento, a pesar de 
que la productividad de los asalariados crece 15 por ciento ${ }^{13}$.

La desregulación y flexibilización del mercado laboral, justificadas como mecanismos para incrementar la productividad y competitividad, implican el abaratamiento de la fuerza de trabajo mientras que el desgaste físico y emocional incrementa la vulnerabilidad de presentar daños a la salud específicos asociados con las actividades que se realizan ${ }^{14,15}$. La terciarización de servicios impacta de manera negativa la estabilidad y las condiciones de bienestar de los trabajadores y sus familias con una afectación directa sobre su nivel de vida, limitando el acceso a una alimentación suficiente y de calidad, a vivienda digna y a servicios de salud y seguridad social. Violaciones a contenidos del derecho al trabajo que el Comité DESC identifica desde 1999 en su informe sobre México, expresando su preocupación por la insuficiencia del salario mínimo para acceder a la canasta básica y la privatización de la seguridad social ${ }^{16}$.

La embestida contra los trabajadores se expresa en el constante deterioro de sus ingresos y sus condiciones laborales que afectan cada vez más a los empleos formales, que sin embargo cuentan con ventajas relativas en comparación de aquellas personas que se encuentran desempleadas o en la informalidad, que no tienen acceso a servicios de la seguridad social, ni al sistema de pensiones y no cuentan con las prestaciones económicas y sociales consideradas en la legislación.

Para el primer trimestre de 2016, 42 por ciento de la población ocupada tuvo ingresos iguales o inferiores a dos salarios mínimos y el 6.3 por ciento no percibió ningún ingreso por su trabajo. Respecto al acceso a instituciones de salud el 62.9 por ciento de los ocupados carece de esta prestación laboral ${ }^{10}$.

Tres de cada 5 mexicanos se desempeñan fuera del mercado formal de trabajo, con ocupaciones y actividades de baja inversión, poco desarrollo tecnológico, condiciones de trabajo no protegidas y sin cotizar a la seguridad social. Entre ellos están los trabajadores por cuenta propia, trabajadores del hogar, los jornaleros agrícolas, la mayor parte de los trabajadores con discapacidad, de los trabajadores indígenas y trabajadores migrantes, con inequidades de género y de condición socioeconómica y dificultades crecientes por las altas tasas de informalidad y las contrataciones de corto tiempo para obtener una pensión ${ }^{17}$.

El informe sobre Panorama Social de América Latina 2015 señala que la pobreza y la indigencia disminuyeron en la mayoría de los países latinoamericanos en el periodo 2010-2014 a excepción de tres, entre ellos México ${ }^{18}$. El informe indica que México ocupa el lugar 13 en pobreza de los 15 países de la región, con un aumento de 2.9 por ciento de la población pobre en el país, incremento atribuido a la erosión salarial componente esencial del funcionamiento del capitalismo mexicano ${ }^{19}$. Este problema se expresa también en el índice de tendencia laboral de la pobreza (ITLP) que presenta una tendencia ascendente desde el $2008^{20}$.

En México, la informalidad expresada como trabajo precario en el sector formal asciende a 28 por ciento de trabajadores en empresas formalmente establecidas ${ }^{21}$ y 6 de cada 10 personas ocupadas laboran en el sector informal. La aprobación de la reforma laboral que legaliza el trabajo precario incrementa esta problemática ${ }^{22}$.

Bajo la medición de informalidad laborar basado en el Measuring Informality: a new statistical manual on the informal sector and informal employment, la Tasa de Informalidad Laboral (TIL) o empleo informal presentaba valores de $59.3 \%$ en el primer trimestre de 2005 , mientras que para el cuarto trimestre del 2015 alcanzó $59.6 \%{ }^{8}$.

Las entidades federativas con menores proporciones de empleo informal (TIL) en 2015 fueron Chihuahua (39.5\%) Coahuila $(40.5 \%)$ y Nuevo León $(41.1 \%)$, mientras que las mayores tasas correspondieron a las entidades con los mayores niveles de pobreza en el país (Oaxaca, 79.9; Chiapas, 78.7; Guerrero 76.8 por ciento $)^{8}$.

Ante el incremento del sector informal, otra dimensión a considerar son los efectos que se tiene respecto a la recaudación fiscal y la participación del Estado en el financiamiento de las instituciones públicas, principalmente las instituciones de educación, salud y seguridad social. Existe una disminución en las fuentes de empleo formales y el constante crecimiento del desempleo y el empleo informal no solo incide directamente en el aseguramiento de los trabajadores y sus familias sino también en el financiamiento de instituciones de seguridad social, principalmente el IMSS ${ }^{23}$.

\section{El derecho a la salud y a la seguridad social}

El derecho a la salud está parcialmente reconocido en el artículo cuarto constitucional que establece que toda persona tiene derecho a la protección de la salud y la ley definirá las bases y modalidades para el acceso a los servicios de salud $^{24}$. 
El reconocimiento de los alcances del Derecho a la Salud y del Derecho a la Protección de la Salud es importante, mientras el primero engloba un conjunto de satisfactores que permiten resolver necesidades humanas interrelacionadas para el logro de la salud reconocidos como determinantes sociales de la salud en la Observación General 14 del PIDESC; el segundo restringe su alcance a lo que los Estados desarrollan respecto a la atención de la salud, básicamente a través de sistemas institucionalizados que promueven la salud, previenen y atienden la enfermedad e incluyen diversas tareas que van desde la rectoría, regulación y normatividad, el financiamiento, organización, producción y prestación de servicios, hasta las modalidades de tutela del derecho y resolución de controversias ${ }^{25}$.

$\mathrm{Si}$ bien en México el texto constitucional se refiere a la protección a la salud, a partir de la reforma sobre derechos humanos realizada en 2011, es válido hablar del Derecho a la salud y del Estado mexicano como sujeto obligado a promover, proteger, respetar y garantizar este derecho en su acepción más amplia. La reforma modificó el artículo $1^{\circ}$ de la Constitución Política de los Estados Unidos Mexicanos, reconociendo a los tratados internacionales en el mismo nivel de jerarquía que la Constitución e incorporando el principio pro homine ${ }^{26}$.

En la Declaración Universal de los Derechos Humanos ${ }^{3}$ y en el Pacto Internacional de los Derechos Económicos, Sociales y Culturales $(\mathrm{DESC})^{4}$, se considera como un derecho humano y el Protocolo Adicional a la Convención Americana sobre Derechos Humanos en materia de Derechos Económicos, Sociales y Culturales, conocido como "Protocolo de San Salvador" señala en su artículo 9 incisos 1 y 2: que toda persona tiene derecho a la seguridad social que la proteja contra las consecuencias de la vejez y de la incapacidad que la imposibilite física o mentalmente para obtener los medios para llevar una vida digna y decorosa, que en caso de muerte del beneficiario, las prestaciones de seguridad social serán aplicadas a sus dependientes; que cuando se trate de personas que se encuentran trabajando, se cubrirá al menos la atención médica y el subsidio o jubilación en casos de accidentes de trabajo o de enfermedad profesional y en el caso de las mujeres gozarán de licencia retribuida por maternidad antes y después del parto ${ }^{27}$. Así mismo, la Organización Internacional del Trabajo (OIT), señala que la seguridad social es una expresión clara de la importancia que una sociedad le otorga a la protección de sus integrantes frente a las con- tingencias económicas y sociales derivadas de la pérdida de ingresos a consecuencia de enfermedades, maternidad, riesgos de trabajo, invalidez, vejez y muerte. La reconoce como un derecho humano fundamental y señala que su desarrollo fortalece la cohesión social, la inclusión y la vida digna $^{28}$.

Desde esta visión, la seguridad social incluye un conjunto de prestaciones, en México, entre las más importantes destacan pensiones, riesgos de trabajo, licencia por maternidad, protección en situaciones de fragilidad, atención médico-sanitaria y apoyos culturales y recreativos. Sin embargo, el sistema de seguridad social, al igual que el de atención a la salud es muy heterogéneo y está vinculado al empleo formal, por lo que su cobertura es limitada.

\section{Cobertura y afiliación al sistema de salud y seguridad social}

En México el sistema institucionalizado de salud y seguridad social es heterogéneo y fragmentado $^{29}$. Está constituido por el sector público y el privado. Las instituciones de salud y seguridad social públicas más importantes son el Instituto Mexicano del Seguro Social (IMSS), Instituto de Seguridad y Servicios Sociales de los Trabajadores del Estado (ISSSTE), Petróleos Mexicanos (PEMEX), Secretaría de la Defensa (SEDENA), Secretaría de Marina (SEMAR), más los regímenes estatales. Estas instituciones se fundamentan en un régimen contributivo de salud y ofrecen seguridad social laboral, que incluye atención médica, pensiones y diversas protecciones a lo largo del curso de vida.

Las instituciones que prestan atención a la población sin seguridad social como el Programa IMSS-Oportunidades (IMSS-O) hoy IMSS-Prospera (IMSS-P) y el Sistema de Protección Social en Salud (SPSS) cuya forma operativa es el Seguro Popular de Salud (SPS) se financian con impuestos generales (federales y estatales) y cuotas de recuperación y reciben recursos económicos para la atención de grupos de bajos ingresos. El sector privado se encuentra conformado por prestadores de servicios que trabajan en consultorios, clínicas y hospitales privados además de la compra de servicios a compañías aseguradoras ${ }^{30}$.

El programa IMSS-O tiene como antecedente al Programa IMSS-Coplamar (1979) y en su versión Oportunidades fue creado para atender a las personas en las zonas de mayor rezago económico y a decir de la OCDE, el programa "... ayuda a los pobres a invertir en capital humano. 
Este programa ha sido muy exitoso. Sin embargo, con el tiempo sería deseable fortalecer el sistema de asistencia social para complementar a Oportunidades" y muchos países lo han tomado como modelo para su implementación ${ }^{31,32}$. A pesar de esta visión optimista, diversos estudios han demostrado que este programa no ha tenido un impacto significativo en la reducción de la pobre$\mathrm{za}^{33}$. La pobreza alimentaria en los hogares que disponen el programa Oportunidades hoy Prospera disminuyó en $2.09 \%$; las familias en pobreza de capacidades, $1.27 \%$, y los hogares en pobreza patrimonial disminuyeron solo $0.46 \%$. Resalta que los resultados son menos evidentes en la región Sur-Sureste del país, la cual se conforma de los estados con mayores niveles de pobreza y rezago en el país ${ }^{34}$.

Los resultados más relevantes del programa se enfocan en el rubro de la educación y el incremento en el uso de los servicios de salud, principalmente de carácter preventivo ${ }^{35}$ pero ello se debe a que son condiciones obligatorias para la permanencia de los individuos y sus familias al programa y puedan acceder a los apoyos económicos.

El Sistema de Protección Social en Salud surge como un mecanismo para el acceso a un seguro médico público dirigido a la población no asalariada y sin acceso a los servicios públicos de seguridad social (autoempleados, desempleados, trabajadores del sector informal de la economía y personas que están fuera del mercado laboral formal). Desde su origen, se consideró implementación del SPSS en tres etapas: la afiliación universal; la cobertura universal, y posteriormente alcanzar la cobertura efectiva universal, que garantiza a todos, de manera igualitaria, el máximo nivel alcanzable de resultados en salud a partir de un paquete de servicios de alta calidad que también evita las crisis financieras a través de la reducción de los gastos de bolsillo ${ }^{36}$. Sin embargo, la cobertura universal a los servicios de salud se ha limitado a la afiliación de la población bajo graves problemas de infraestructura y recursos humanos, limitando el concepto de cobertura universal al acceso regular a un paquete de servicios de salud para las personas inscritas al programa ${ }^{37}$. Sin embargo, el objetivo de disminuir el gasto de bolsillo no se ha logrado, por lo contrario, se ha incrementado de manera consistente (Figura 1) aun cuando se justifica dicho problema a la falta de autonomía de los prestadores para ofertar servicios de calidad y así disminuir el gasto de bolsillo ${ }^{38}$, lo que puede incentivar el mercado privado.
En el periodo 1990-2011 se aprecia un cambio sustantivo en la estructura del gasto: en 1990 el destinado a la población con seguridad social representaba el 81.7 por ciento de total de gasto público en salud, para 2011 esta proporción disminuye a 55.7 puntos y crece significativamente la participación del gasto destinado a la población sin seguridad social, alcanzando 44.3 puntos porcentuales $^{39}$. También se reduce la inversión pública en equipo e infraestructura sanitaria, se alienta la subrogación y los proyectos público-privados de prestación de servicios ${ }^{40}$.

La reducción del financiamiento público en seguridad social tuvo efectos negativos sobre los indicadores convencionales de disponibilidad de servicios, que hoy son menores que hace tres décadas. En forma paralela se privatizó el sistema de pensiones de la seguridad social, que pasó de un sistema de reparto a uno de capitalización individual, creándose las Administradoras de Fondos para el Retiro (AFORES) y se estableció la garantía de pensión mínima.

La afiliación a los servicios de salud ha sufrido importantes modificaciones, el SPS ha incrementado su cobertura hasta lograr en 2015 cerca del $42 \%$ de la población asegurada mientras que los sistemas de seguridad social, instituciones que protegen a los trabajadores insertos en el mercado laboral formal, ha reducido el porcentaje de población asegurada con una tendencia similar a la observada en la distribución del gasto total por instituciones de salud (Figuras 2 y 3 ).

\section{Barreras de acceso}

Debido a las características del sistema de salud mexicano, existe un acceso inequitativo a los servicios públicos de salud determinados por la condición laboral de las personas. La atención se encuentra claramente diferenciada, mientras que los servicios de seguridad social proveen atención médica según las necesidades de atención de las personas de manera amplia, limitadas por los recursos crecientemente escasos y reguladas por los tiempos de espera, las personas que se encuentran fuera del mercado laboral formal tiene como opción la atención de servicios privados según su capacidad de pago, el autocuidado, la automedicación o la afiliación al SPS.

Se ha señalado que el SPS presenta diversas características regresivas como la visión restrictiva del derecho a la protección a la salud al ofrecer un paquete de intervenciones predominantemente curativas y costo-efectivas; se subordina la universalidad y la gratuidad a criterios basa- 


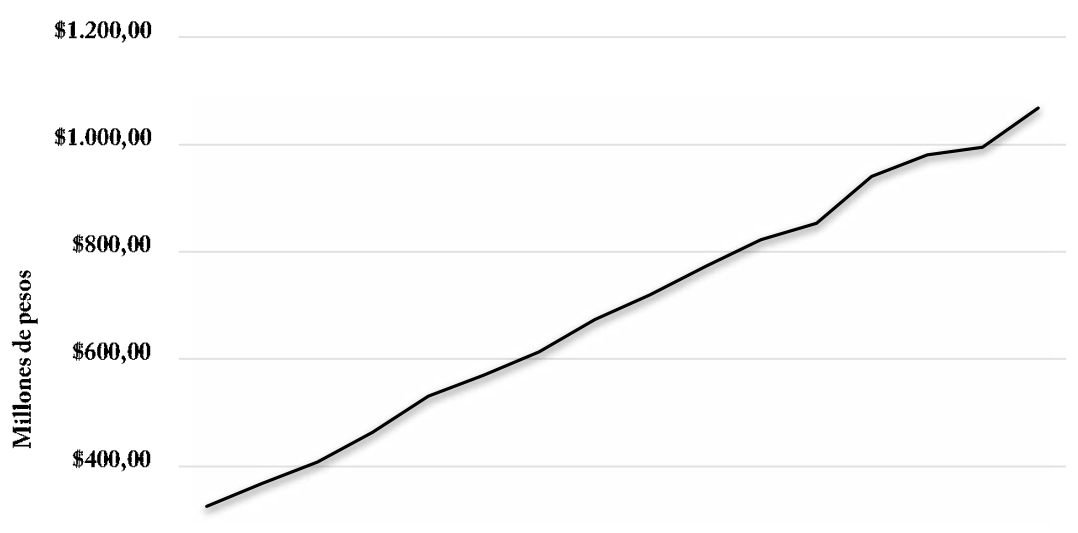

$\$ 200,00$

$\$ 0,00$

2000200120022003200420052006200720082009201020112012201320142015

Año

Figura 1. Gasto de bolsillo en salud 2000-2015, México.

Realización propia a partir de información disponible de la Dirección General de Información en Salud (DGIS). Base de datos de cuentas en salud a nivel federal y estatal, 1990-2014. [en línea]: Sistema Nacional de Información en Salud (SINAIS).

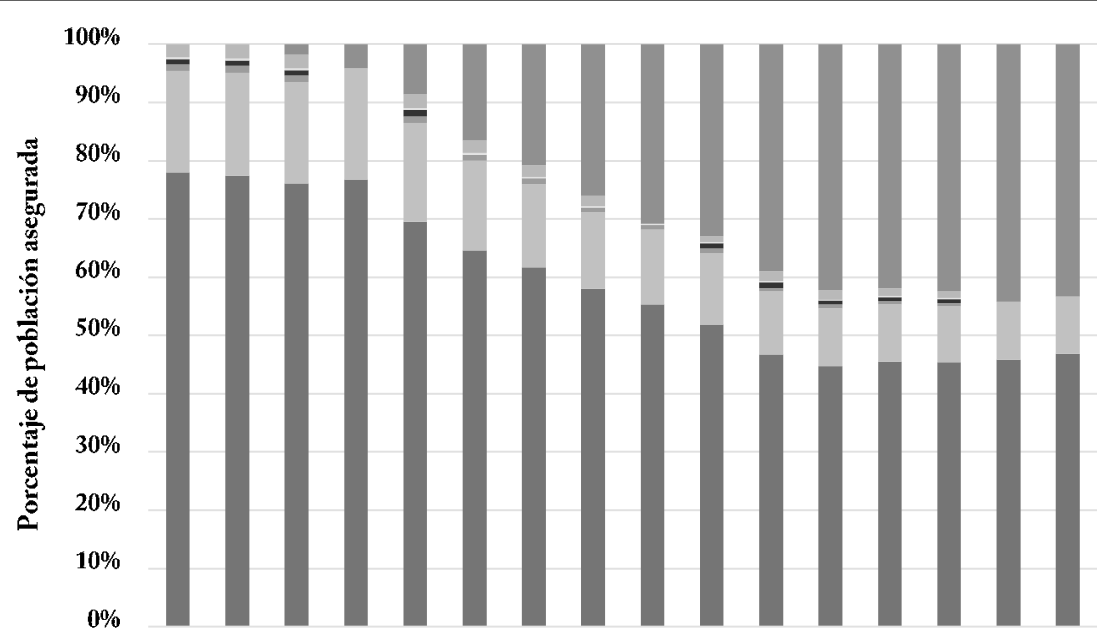

2000200120022003200420052006200720082009201020112012201320142015

Año

$\square$ IMSS ISSSTE PEMEX $\square$ SDN SM Servicios Estatales $\square$ Seguro Popular

Figura 2. Distribución de población asegurada por institución de salud, 2000-2015.

Fuente: Realización propia a partir de datos disponible de la Dirección General de Información en Salud (DGIS). Base de datos de cuentas en salud a nivel federal y estatal, 2000-2015. En el año 2015 y 2015 no hay datos disponibles para los servicios estatales, Marina, Defensa y PEMEX. 


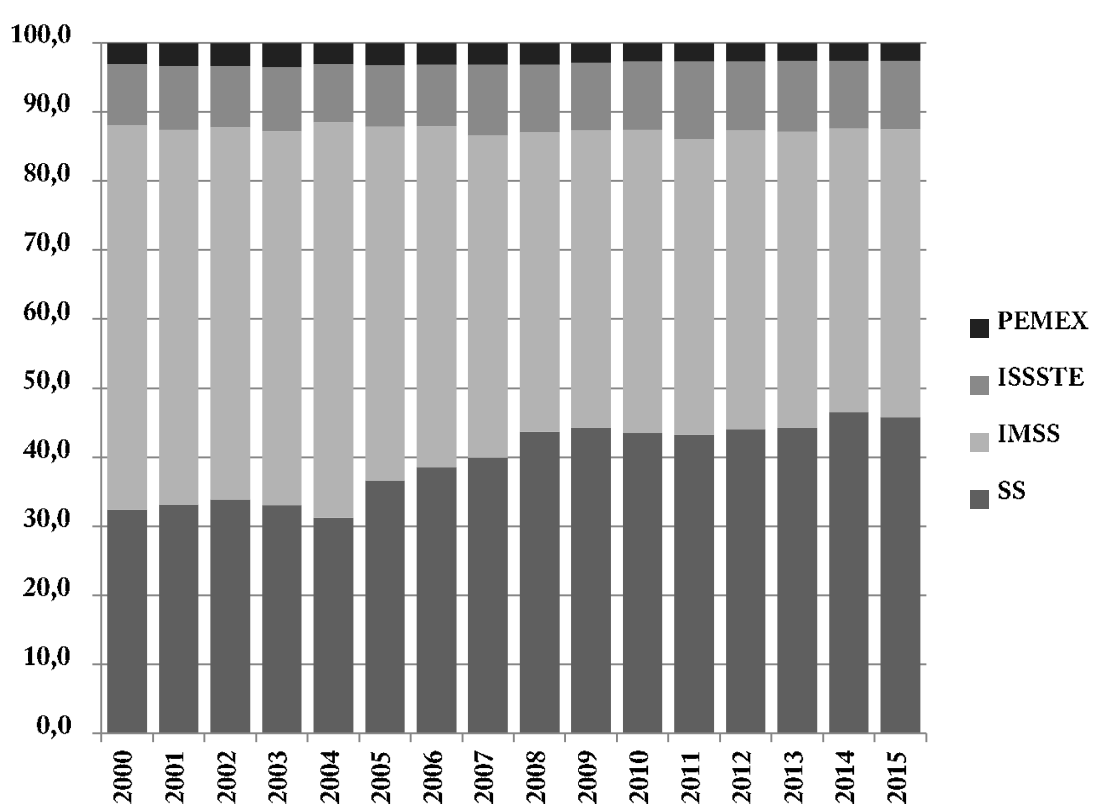

Figura 3. Gasto total en salud por institución, México 2000-2015.

Fuente: Realización propia a partir de datos disponible de la Dirección General de Información en Salud (DGIS). Base de datos de cuentas en salud a nivel federal y estatal, 2000-2015.

dos en las condiciones socioeconómicas de los usuarios; el esquema de aseguramiento del SPS, limita las posibilidades de compartir riesgos, de transferir recursos de "ricos a pobres" o de sanos a enfermos, y aleja la posibilidad de instaurar un esquema de aseguramiento solidario, por lo que el programa se enfrentará a la insostenibilidad financiera ${ }^{41}$.

A pesar de sus limitaciones, el SPS ha resultado útil para minar las propuestas de universalizar la seguridad social y presentar a la seguridad social laboral como un privilegio y no como el resultado de tres derechos en estrecha interdependencia: el derecho al trabajo, el derecho a la salud y el derecho a la seguridad social.

\section{Reflexiones finales}

La embestida contra los trabajadores se expresa en el constante deterioro de sus ingresos y sus condiciones laborales afectadas de diversas formas tanto en empleos formales que se precarizan como en trabajos en la economía informal. El desempleo, el subempleo, el deterioro salarial y el desmantelamiento del sistema público de protección social, aunado a la privatización del siste- ma de pensiones conforman un contexto adverso para la garantía de derechos.

La estructuración de la seguridad social laboral es profundamente heterogénea y en particular las dos instituciones con mayor cobertura (IMSS e ISSSTE), que además de ofrecer servicios sanitarios para su población derechohabiente también incorporaban prestaciones económicas, sociales y culturales son resultado del periodo histórico y sociopolítico en el que se impulsó la industrialización del país y la integración nacional a través de instituciones que respondían a la vertiente de bienestar del Estado mexicano.

El IMSS-P y el SPS responden a otro momento de la historia del país, modelado por la primacía del mercado, la liberalización y la flexibilización laboral que se traduce en el campo social en políticas asistenciales para la población que no tiene empleo formal y por tanto carece de acceso a sistemas de seguridad social. Para esta población se estructura un SPSS que ofrece algunos servicios sanitarios costo efectivos ordenados en las lógicas de focalización de poblaciones y selectividad de intervenciones definiendo trabajadores de "primera" y de "segunda" clase debido a la falta de un sistema de seguridad social real que integre los diferentes mercados laborales ${ }^{42}$. 
Este sistema de salud y seguridad social, heterogéneo y fragmentado, configura distintas ciudadanías sociales, contribuye a la diferenciación por condición laboral y divide a los trabajadores, dificultando la defensa colectiva y unificada del derecho al trabajo, el derecho a la salud y el derecho a la seguridad social.

Los impactos directos en salud siguen siendo diferenciados, a pesar de una disminución en el financiamiento y adscripción en los sistemas de seguridad social, estos siguen teniendo una ventaja relativa respecto a quienes se afilian al Seguro Popular o no tienen derechohabiencia ${ }^{43}$. Los motivos son diversos y no es una relación directa y simple, por el contrario, muestran la compleja relación entre las condiciones de vida y la relación con el trabajo que determinan daños de salud diferenciados entre las poblaciones y que, además, la inserción laboral condiciona al tipo de atención médica a la cual acceder.

Ante este panorama de precarización de los servicios públicos de salud, específicamente de los sistemas de seguridad social, la definición de un nuevo modelo de atención en la población mexicana a corto plazo se dirige a la implementación un sistema de portabilidad para la atención médica y al intercambio de servicios entre instituciones independientemente de la afiliacióndel usuario. Sin embargo, se trata de respuestas muy limitadas frente al desafío de ampliar el mercado laboral formal, recuperar la capacidad adquisitiva del salario e impulsar un sistema universal de salud y seguridad social tomando como plataforma institucional al IMSS ${ }^{44}$. Este sistema es fundamental para avanzar en la justicia social, para lograrlo, es imprescindible crear puestos de trabajo formales y una recaudación fiscal progresiva y equitativa.

Finalmente, es importante señalar que el Estado mexicano debe retomar su obligación de garantizar los derechos individuales y colectivos, incluyendo los derechos al trabajo digno, a la salud y a la seguridad social para mejorar la vida de los trabajadores.

\section{Colaboradores}

O López-Arellano trabajó en la concepción teórica y edición final y O Medina-Gómez en la investigación, metodología, análisis y redacción. 


\section{Referencias}

1. De la Garza E. Neoliberalismo y Estado. In: Laurell A. Estado y Políticas Sociales en el Neoliberalismo. México: UAMX; 1994. p. 59-73.

2. Vite MA. Estado, globalización y exclusión social. Política y cultura 2006; 25:9-26.

3. Ordoñez G. El estado social en México. Un siglo de reformas hacia un sistema de bienestar excluyente. México: El Colegio de la Frontera Norte/XXI Editores; 2017.

4. Organización de Naciones Unidas (ONU). Declaración Universal de Derechos Humanos. Resolución de la Asamblea General 217 A (iii), del 10 de diciembre; 1948.

5. Organización de Naciones Unidas (ONU). Pacto Internacional de Derechos Económicos, Sociales y Culturales. A/RES/2200 A (XXI) del 16 de diciembre, Nueva York: ONU; 1966.

6. CDESC (2000). Observación General 14. El derecho al disfrute del más alto nivel posible de salud. Ginebra: Comité de Derechos Económicos, Sociales y Culturales, ONU. Disponible en: http://wwwl.umn.edu/humanrts/gencomm/epcomm 14s.htm

7. Constitución Política de los Estados Unidos Mexicanos (2014). Título Primero. Cap. I. De los derechos humanos y sus garantías. [acceso 2017 Mar 18]. Disponible en: http://www.diputados.gob.mx/LeyesBiblio/htm/1.htm

8. Organización Internacional del Trabajo (OIT). La economía informal y el trabajo decente: una guía de recursos sobre políticas, apoyando la transición hacia la formalidad. Ginebra: OIT; 2013.

9. Instituto Nacional de Estatística e Geografia (INEGI). Encuesta Nacional de Ocupación y Empleo. Informalidad laboral. Tasas de informalidad trimestral. México: INEGI; 2015.

10. Observatorio de política social y derechos humanos (OPSDH). El trabajo en México. Un acercamiento desde la perspectiva de derechos humanos. México: Incide Social; 2012.

11. Guillén A. Crisis inmobiliaria y la recesión global. En: Coloquio América Latina: escenarios del nuevo siglo; Buenos Aires. Oct 2008.

12. Ortiz E. Los nuevos escenarios económicos de la crisis. En: De la Garza E, coordinador. La situación del trabajo en México. México: Plaza y Valdez; 2012. p. 15-38.

13. Camberos M, Bracamontes J. La caída de los salarios en México 1980-2010: la productividad y la informalidad como explicación. Equilibrio económico. Revista de Economía, Política y Sociedad 2015; 11(40):169-194.

14. Ibarra M, González L. La flexibilidad laboral como estrategia de competitividad y sus efectos sobre la economía, la empresa y el mercado de trabajo. Contaduría y Administración 2010; 231:33-52.

15. Medina O. Prevalencia de mobbing en trabajadores y factores de riesgo asociados. Gaceta Médica de México 2016; 152:452-456.
16. Organización de las Naciones Unidas (ONU). Re copilación de las observaciones generales y recomendaciones generales adoptadas por órganos creados en virtud de tratados de derechos humanos. Nueva York: ONU; 2008. (HRI/GEN/1/Rev.9/Vol.I), 27 de mayo. [acceso 2017 Mar 18]. Disponible en: http://daccessdds-ny.un.org/doc/UNDOC/GEN/G08/422/38/PDF/ G0842238.pdf?OpenElement, consultado el 18 de marzo de 2017.

17. Wissing T. Los pisos de protección social como elemento constitutivo del trabajo decente. Seguridad social en salud. Pensiones y desempleo. Miradas desde México y América Latina. Senado de la República LXIII Legislatura, Comisión de Seguridad Social. México, pp. 45-66

18. CEPAL (2016). Panorama Social de América Latina 2015. [acceso 2017 Mar 18]. Disponible en: http:// repositorio.cepal.org/bitstream/handle/11362/39965/ S1600227_es.pdf?sequence $=1$

19. Delgado O. (2016). Datos y resultados en el registro de la pobreza. La Jornada. [acceso 2017 Mar 18]. Disponible en: http://www.jornada.unam. $\mathrm{mx} / 2016 / 03 / 24 /$ opinion/026aleco

20. CONEVAL (2017). [acceso 2017 Mar 18]. Disponible en: http://www.coneval.org.mx/Medicion/Paginas/ ITLP-IS_resultados_a_nivel_nacional.aspx

21. Observatorio de Política Social y Derechos Humanos (OPSDH). Informe alternativo de sociedad civil al primer informe de México al Protocolo de San Salvador. 2014. [acceso 2017 Mar 18]. Disponible en: http://observatoriopoliticasocial.org/wordpress/wp-content/ uploads/2014/06/Informe-Alternativo-PSS.-México. pdf

22. México. Ley Federal del Trabajo. Diario Oficial de la Federación 2015; 12 jun.

23. Laurell A, Herrera J. La Segunda Reforma de Salud: Aseguramiento y compra-venta de servicios. Salud Colectiva 2010; 6(2):137-148.

24. México. Decreto por el que se adiciona con un párrafo penúltimo el artículo $4^{\circ}$ de la Constitución Política de los Estados Unidos Mexicanos. Diario Oficial de la Federación 1983; 03 mar.

25. López O, Medina O, Blanco J. Derecho A la Salud, Desigualdades SocioSanitarias y Políticas de Salud en la Ciudad de México. Evalúa-DF. [Internet]. 2011. [citado 2017 Mar 6]. Disponible en: http://www.evalua df.gob.mx/files/transparencia/2011/dsalud.pdf

26. México. Decreto por el que se modifica la denominación del Capítulo I del Título Primero y reforma diversos artículos de la Constitución Política de los Estados Unidos Mexicanos. Diario Oficial de la Federación 2011; 10 jun. [citado 2017 Mar 1]. Disponible en: http://www.dof.gob.mx/nota_detalle.php?codigo $=5194486 \&$ fecha $=10 / 06 / 2011$

27. Organización de Estados Americanos (OEA). Protocolo Adicional a la Convención Americana sobre Derechos Humanos en materia de Derechos económicos, Sociales y Culturales, conocido como Protocolo de San Salvador. San Salvador: OEA; 1988. 
28. Organización Internacional del Trabajo (OIT). Seguridad Social para Todos. Ginebra: OIT; 2011.

29. Laurell A. La reforma contra la salud y seguridad social. México DF: ERA; 1997.

30. Gómez-Dantés O, Sesma S, Becerril V, Knaul F, Arreola H, Frenk J. Sistema de salud de México. Salud Pública de México 2011; 53(Supl. 2):s220-s232.

31. OCDE, Estudios económicos de la OCDE: México 2011. [Internet]. 2012 [citado 2017 Mar 1]. Disponible en: http://dx.doi.org/10.1787/9789264115934-es

32. Banco Mundial. México y Colombia exportan sus éxitos en transferencias condicionadas de efectivo. [Internet]. 2012 [citado 2017 Mar 1]. Disponible en: http:// www.bancomundial.org/es/news/feature/2012/07/31/ mexico-colombia-exportan-exitos-en-transferencias-condicionadas-de-efectivo

33. Cortés F, Banegas I, Solís P. Pobres con oportunidades: México 2002-2005. Estudios Sociológicos 2007; 25(73):3-40.

34. Bracamontes J, Camberos M. La pobreza en México y sus regiones: un análisis del impacto del programa Oportunidades en el periodo 2002-2006. Papeles de Población 2011; 17(67):135-175.

35. Bertozzi S. Impacto de Oportunidades en la utilización de servicios de salud. Salud Pública de México 2007; 49 (Supl. 1):199-201.

36. Gómez-Dantés O, Ortiz M. Seguro Popular de Salud: siete perspectivas. Salud Pública de México 2004; 46(6):585-588

37. Laurell A, Herrera J. La Segunda Reforma de Salud: Aseguramiento y compra-venta de servicios. Salud Colectiva 2010; 6(2):137-148.

38. González-Block MA, Figueroa A, García-Téllez I, Alarcón J. Asignación financiera en el Sistema de Protección Social en Salud de México: retos para la compra estratégica. Salud Pública de México 2016; 58(5):522-532.
39. Ponce C. Gasto Público en Salud. El caso de México. OIAPSS, 2014. [citado 2017 Mar 1]. Disponible en: http://www.oiapss.org/wp-content/uploads/2014/12/ GPS.ARTICULO_mexico.pdf

40. Herrera J, Laurell C. Proyectos de prestación de servicios. Una nueva forma de privatización. In: Peña F, Parra B, coordinadores. La medicina social en México II. Globalización neoliberal. México: ALAMES-MX/ Ed. Eón/ENAH; 2010. p. 163-175.

41. Laurell A. Los seguros de salud mexicanos: cobertura universal incierta. Cien Saude Colet 2011; 16(6):27952806.

42. Noriega M, Franco J, Montoya A, Garduño A, León L, Martínez S, Cruz A. Deterioro de las condiciones de trabajo y de salud en México. Salud de los Trabajadores 2009; 17(1):61-68.

43. López O, Rivera JA. Sistema Nacional de Salud Universal: universalidad de mercado y restricción de derechos. In: Valencia E, Barba C. Nueva ronda de reformas estructurales ¿nuevas politicas sociales? México: UIA/ ITESO; 2016. p. 277-298.

44. López O, Jarillo E. ¿ Hacia dónde debe ir el sistema de salud en México? Una perspectiva desde el derecho a la salud. Medicina Social 10(1):1-13.

Artigo apresentado em 08/06/2017

Aprovado em 19/10/2017

Versão final apresentada em 21/10/2017 